\title{
A NOTE ON THE FREE SURFACE INDUCED BY A SUBMERGED SOURCE AT INFINITE FROUDE NUMBER
}

\author{
A. C. KING ${ }^{1}$ AND M. I. G. BLOOR ${ }^{2}$
}

(Received 21 September 1987; revised 2 November 1987)

\begin{abstract}
The free surface due to a submerged source in a fluid of finite depth at infinite Froude number is reconsidered. A conformal transformation technique is used to formulate this problem as an integral equation for the free-surface angle. An elementary solution is found for the equation, which results in a closed form expression for the free-surface elevation. Comparison is made with previous numerical solutions.
\end{abstract}

\section{Introduction}

The classical problem of the free-surface flow of an ideal fluid induced by a submerged source or sink with gravity as the restoring force has been the subject of many papers. Solutions which have a stagnation point directly above the source or sink have been given by Havelock [4], Peregrine [7] and by VandenBroeck, Schwartz and Tuck [10]. More recently there have been several papers dealing with these flows in which it has been assumed that a cusp exists in the free surface directly above the source or sink. Tuck and Vanden-Broeck [8] appear to have been the first to have encountered this type of solution in classical free-surface flow when they considered the flow due to a submerged source in a fluid of infinite depth. These authors found numerically that a cusped solution existed at the unique Froude number 1.776; no cusped solutions were found at other Froude numbers. This work was generalized by Hocking

\footnotetext{
${ }^{1}$ Department of Theoretical Mechanics, University of Nottingham, Nottingham, England

${ }^{2}$ School of Applied Mathematical Studies, University of Leeds, Leeds, England

(C) Copyright Australian Mathematical Society 1988, Serial-fee code 0334-2700/88
} 
[5], who considered flows induced by a source at the apex of a submerged semiinfinite triangular mountain. Cusped solutions were found numerically with a unique Froude number corresponding to each apex angle of the triangular mountain. Collings [3] has considered the flow due to a line source or sink within a fluid of finite depth and above a horizontal bottom, but with no restoring force. Hodograph methods were used to find cusped solutions when the source/sink was in two positions: on the flat bottom (which is a limiting case of Hocking's work), and when the source depth was 0.56742 of the far fluid depth. This last figure arose as a result of numerical experimentation which was necessary to give the correct flux of fluid at infinity. Cusped flows due to a sink within a fluid of finite depth, with gravity acting, have been considered by Vanden-Broeck and Keller [9]. These authors have numerically confirmed the solutions of Collings and have also shown that cusped flows exist for all Froude numbers larger than some particular value.

Very recently, Hocking [6] has reconsidered the problem of Collings and, by using the analytical properties of the complex potential, has derived an integral equation satisfied by the free-surface angle; no closed-form solution to this equation is found. To progress, Hocking transforms his equation into the standard aerofoil equation for which a formal solution, containing an integral, exists. This integral is then evaluated by trapezoidal discretisation to give a numerical solution for the free-surface angle; some further computation gives the free-surface elevation. To provide a check on the numerical scheme, Hocking also derives a series solution to the integral equation. The radius of convergence of this series was shown to be unity, which limited it validity to cases when the source or sink is close to the flat bottom. A comparison of the numerical and series results for the cusp depth was made, and these showed excellent agreement provided the source was not too far from the bottom. The numerical results presented show solutions to exist over a variety of depths for the source or sink within the fluid and there seems to be no 'critical depth' as in the work of Collings.

An alternative to the classical hodograph methods for free-surface flows has been developed and used by Bloor [1] for periodic water waves, and by Bloor and King [2] for free-surface flows over a stepped bottom. The technique consists of direct conformal transformation of the physical plane onto a half-plane in which the complex potential can be written down. The application of the free-surface condition results in an integral equation, the solution of which enables such quantities as the free-surface elevation to be calculated. This note now considers the same problem as that considered by Collings and Hocking, but the analysis is performed by direct conformal transformation. Explicit closed-form results are found for the equation of the free surface and the cusp height; the numerical and asymptotic results of Collings and Hocking are confirmed as particular cases of these results. 


\section{Mathematical analysis}

A point source of strength $m$ is situated on or above a flat horizontal bottom and beneath a free surface; there is no restoring force. A Cartesian coordinate system $X, Y$ is situated on the flat bottom; the source is a distance $d$ above the origin of these coordinates. A cusp in the free surface is assumed to lie directly above the source. Far away from the source the flow is uniform with speed $U$, directed away from the source; the depth of the fluid is $h$. The usual assumptions of ideal fluid flow allow the dynamics of the flow to be described by an analytic complex potential $W=\Phi+i \Psi$, where $\Phi$ and $\Psi$ are velocity potential and stream function respectively. Boundary conditions appropriate to the free surface are that the fluid speed is constant and equal to $U$ on the free surface. Because of the steady nature of the flow, $\Psi$ is constant on the free surface and the flat bottom. Flux considerations give $\pi m=U h$, and we choose $\Psi=0$ on the free surface, and $\Psi=U h$ on the flat bottom.

The physical problem here is non-dimensionalised using the transformations

$$
z=x+i y=(X+i Y) / h, \quad w=\varphi+i \psi=(\Phi+i \Psi) / U h
$$

As the flow is symmetric about the $y$-axis, it is sufficient to consider the flow to the left of the source. The non-dimensional flow domain and associated boundary conditions are shown in Figure 1. The source is situated at the point $S$, which is at a height $\delta$ above the origin of the coordinates and, due to the nondimensionalisation, its strength is now $1 / \pi . A B S$ is a streamline on which $\psi=1$, $D C S$ is a streamline on which $\psi=0$. The fluid speed $q$ on the free surface is unity, as are the depth and speed of the uniform flow at infinity.

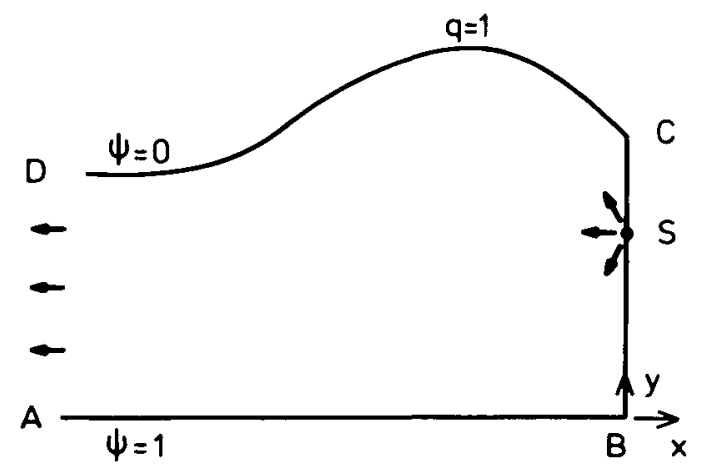

FIGURE 1. The dimensionless flow induced by a submerged source 


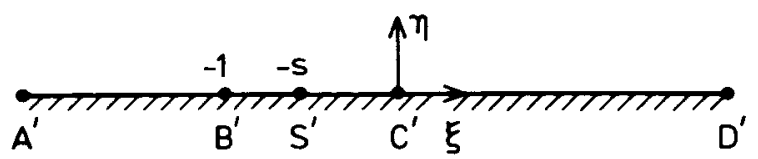

FIgURE 2. The transform $\xi$-plane and the images of the boundary points in the physical $z$-plane

The region occupied by the fluid is now transformed onto the upper half of the $\xi=\varsigma+i \eta$ plane by the mapping

$$
\frac{d z}{d \xi}=\frac{K}{(\xi+1)^{1 / 2} \xi^{1 / 2}} \exp \left\{-\frac{1}{\pi} \int_{t=0}^{\infty} \frac{\theta(t) d t}{\xi-t}\right\} .
$$

The transform plane is shown in Figure 2. In this mapping, which is a generalisation of the Schwarz-Christoffel formula, the quantity $\theta(t)$ is the angle made by the tangent to the free surface with the $x$-axis at the point which corresponds to $\xi=t+i 0$. When $\xi$ is real and positive, the integral in (2.2) becomes a principal value together with a contribution $i \theta(\varsigma)$ which ensures $d z / d \xi$ is an analytic function whose natural logarithm has $\theta(\varsigma)$ as its imaginary part on the positive real axis. It is convenient to write

$$
P=-\frac{1}{\pi} f_{t=0}^{\infty} \frac{\theta(t) d t}{\zeta-t}
$$

where $f$ denotes the Cauchy principal value of the integral. Then on the free surface $s>0$,

$$
\frac{d z}{d \varsigma}=\frac{K}{(\varsigma+1)^{1 / 2} \varsigma^{1 / 2}} \exp \{P+i \theta\} .
$$

In the uniform flow far away from the source, the depth of the fluid is unity. Thus by integrating (2.2) along a vertical line from $A$ to $D$ in the $z$-plane, that is from $A^{\prime}$ to $D^{\prime}$ around a large semi-circle in the $\xi$-plane, it can be seen that $K=-1 / \pi$. The complex potential in the $\xi$-plane is that of a source at the point $-s$ of strength $1 / \pi$, giving the complex velocity on the free surface as

$$
u-i v=q \exp \{-i \Theta\}=\frac{(\varsigma(\varsigma+1))^{1 / 2}}{(\varsigma+s)} \exp \{-P-i(\theta-\pi)\},
$$

where $u$ and $v$ are the $x$ and $y$ components of the fluid velocity, and $\theta$ is the angle made by the fluid velocity with the $x$-axis. From (2.4) we see that

$$
\Theta=\theta-\pi, \quad q=\frac{(\varsigma(\varsigma+1))^{1 / 2}}{(\varsigma+s)} \exp \{-P\} .
$$

Equation (2.5a) shows that the flow is tangential to the free surface and directed away from the source; $(2.5 \mathrm{~b})$ allows the constant velocity condition on the free surface to be written

$$
\frac{1}{\pi} f_{t=0}^{\infty} \frac{\theta(t) d t}{\varsigma-t}=\log \left\{\frac{\varsigma+s}{(\varsigma(\varsigma+1))^{1 / 2}}\right\} \quad 0<\varsigma<\infty
$$


This linear equation for $\theta$ is of Wiener-Hopf form. It is convenient to convert it into a convolution equation over the whole real line by using the change of independent variable $r=-1 / \pi \log \varsigma, \theta(\varsigma)=\tilde{\theta}(r)$ to give

$$
f_{t=-\infty}^{\infty} \frac{\tilde{\theta}(t) d t}{e^{-\pi(r-t)}-1}=\log \left\{\frac{\left(e^{-\pi r}+s\right)}{\left(e^{-\pi r}\left(e^{-\pi r}+1\right)\right)^{1 / 2}}\right\} \quad-\infty<r<\infty .
$$

Equation (2.7) is readily solved in operational form by Fourier transforms. The solution can be written in integral form as

$$
\tilde{\theta}(r)=f_{t=-\infty}^{\infty} \frac{1}{e^{\pi t / 2}-e^{-\pi t / 2}} \log \left\{\frac{\left(e^{-\pi(r-t)}+s\right)}{\left(e^{-\pi(r-t)}\left(e^{-\pi(r-t)}+1\right)\right)^{1 / 2}}\right\} d t .
$$

The integral in (2.8) is evaluated by returning the $\varsigma$-variable, differentiating under the integration sign to produce an integrand which is suitable for contour integration. The resulting expression $\theta^{\prime}(\varsigma)$ is then integrated with the boundary condition $\theta(\infty)=0$ applied to give

$$
\theta(\varsigma)=-\pi / 2+2 \tan ^{-1}\left(\varsigma^{1 / 2} / s^{1 / 2}\right)-\tan ^{-1}\left(\varsigma^{1 / 2}\right) .
$$

The values of $P$ and $\theta$ can now be analytically continued into the upper half of the $\xi$-plane to give the value of the integral appearing in the transformation (2.2) which can now be written in the form

$$
\frac{d z}{d \xi}=\frac{i\left\{\left[\left(2 s^{1 / 2}-1\right) \xi+s\right]+i\left[\xi+2 s^{1 / 2}-s\right] \xi^{1 / 2}\right\}}{(\xi+s)^{2}(\xi+1)^{1 / 2}} .
$$

Equation (2.10) can be integrated, subject to the condition that $z \rightarrow-\infty+i$ as $\varsigma \rightarrow \infty$ along the real axis, to give

$$
\begin{aligned}
& z=2 i+\frac{i}{\pi(1-s)^{3 / 2}}\left\{\frac{2\left(s^{1 / 2}-1\right)(1-s)^{1 / 2}(\xi+1)^{1 / 2}\left\{s+i s^{1 / 2} \xi^{1 / 2}\right\}}{(\xi+s)}\right.+\left(2 s^{1 / 2}-1-s^{3 / 2}\right)\left[\log \left\{\frac{(\xi+1)^{1 / 2}-(1-s)^{1 / 2}}{(\xi+1)^{1 / 2}+(1-s)^{1 / 2}}\right\}\right. \\
&\left.-2 i \tan ^{-1}\left\{\frac{(1-s)^{1 / 2} \xi^{1 / 2}}{s^{1 / 2}(\xi+1)^{1 / 2}}\right\}\right] \\
&\left.+i(1-s)^{3 / 2} \log \left\{\frac{\xi^{1 / 2}+(\xi+1)^{1 / 2}}{\xi^{1 / 2}-(\xi+1)^{1 / 2}}\right\}\right\} .
\end{aligned}
$$

The relation between the source position in the physical and transform planes is found from $i \delta=z(-s)$ and is calculated from (2.11) after some limiting processes to be

$$
\delta=\frac{1}{2}-\frac{1}{\pi} \tan ^{-1}\left\{\frac{2 s-1}{2 s^{1 / 2}(1-s)^{1 / 2}}\right\}-\frac{\left(1-s^{1 / 2}\right)}{\pi(1-s)^{1 / 2}}+\frac{\left(2 s^{1 / 2}-1-s^{3 / 2}\right) \log s}{\pi(1-s)^{3 / 2}} .
$$

Equations (2.11) and (2.12) are the solution to the problem addressed by this note. A graph of the variation of $s$ with $\delta$ is given in Figure 3, and shows that 


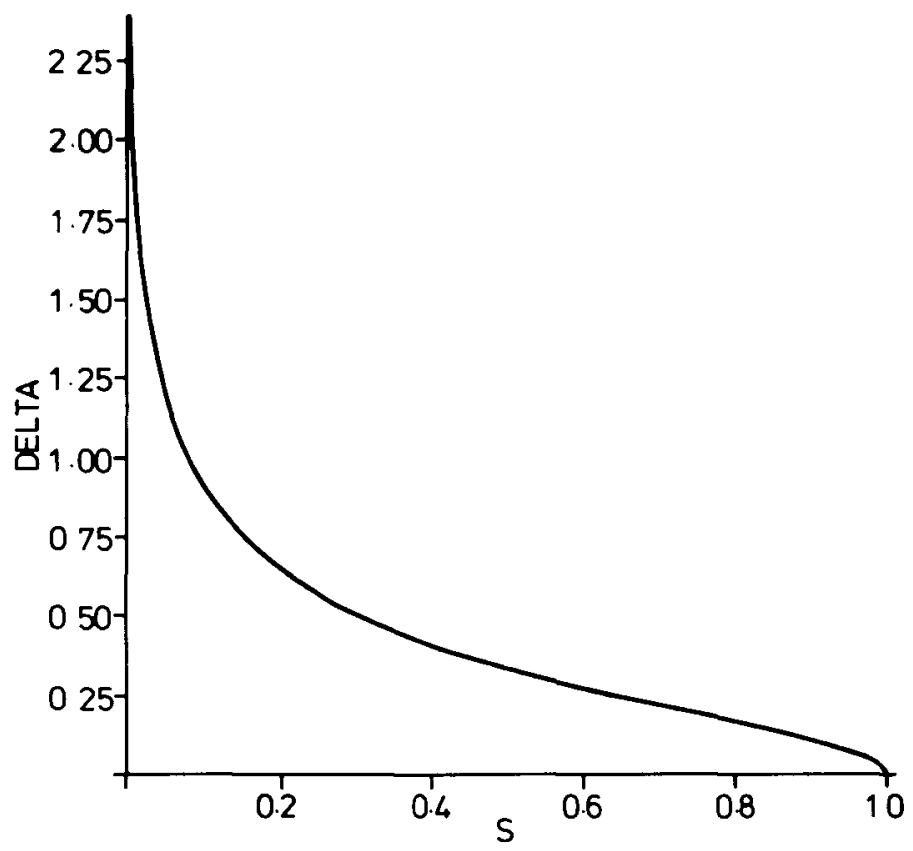

FIGURE 3 . The relation between $\delta$ and $S$

solutions exist for all $\delta \geq 0$, that is, all source positions within the fluid. The equation of the free surface is found by putting $\xi$ real and positive in (2.11). Graphs of the free surface for various positions of the source are given in Figure 4. The position of the cusp in the free surface is $z(0)$ i.e.

$$
\begin{aligned}
y_{\text {cusp }}= & 1+\frac{1}{\pi(1-s)^{3 / 2}}\left\{2\left(s^{1 / 2}-1\right)(1-s)^{1 / 2}\right. \\
& \left.+\left(2 s^{1 / 2}-1-s^{3 / 2}\right) \log \left\{\frac{1-(2-s)^{1 / 2}}{1+(1-s)^{1 / 2}}\right\}\right\}
\end{aligned}
$$

The special case of the source on the bottom is found by letting $s \rightarrow 1$ in (2.11), (2.12) and (2.13) to give the free surface as

$$
z=i+\frac{2}{\pi}\left\{\left(\frac{\varsigma}{\varsigma+1}\right)^{1 / 2}-\sinh ^{-1}\left(\varsigma^{1 / 2}\right)-\frac{i}{(\varsigma+1)^{1 / 2}}\right\}
$$

with $\delta=0$ and $y_{\text {cusp }}=1-2 / \pi$, which is in agreement with the asymptotic result of Collings [6] and the series result of Hocking [10]. Some comparisons of these exact results with the numerical results of Hocking are shown in Figure 


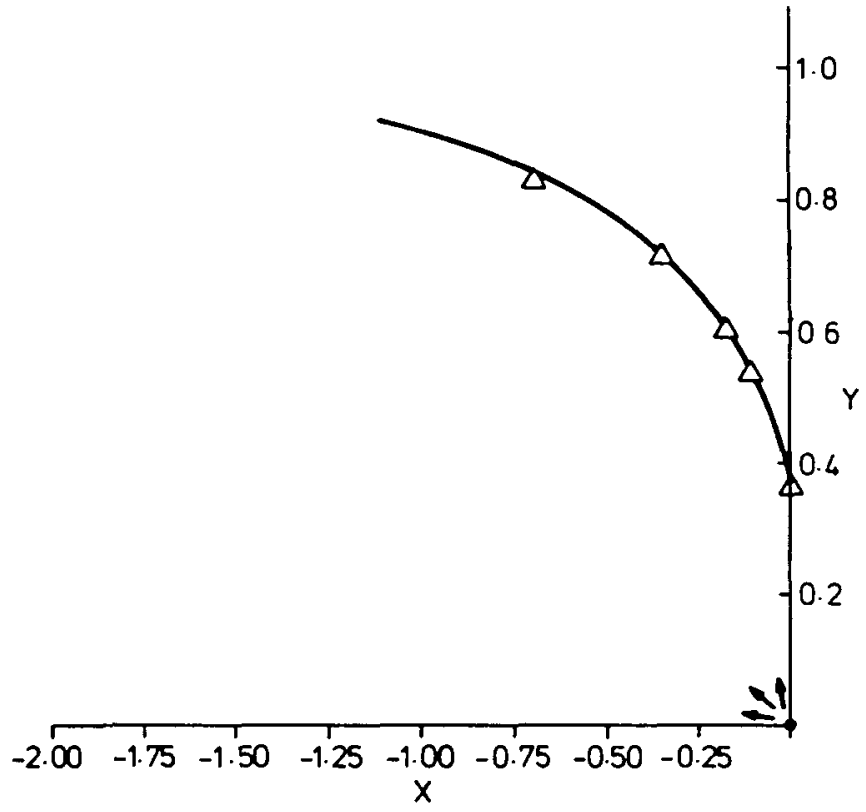

Figure 4(a)

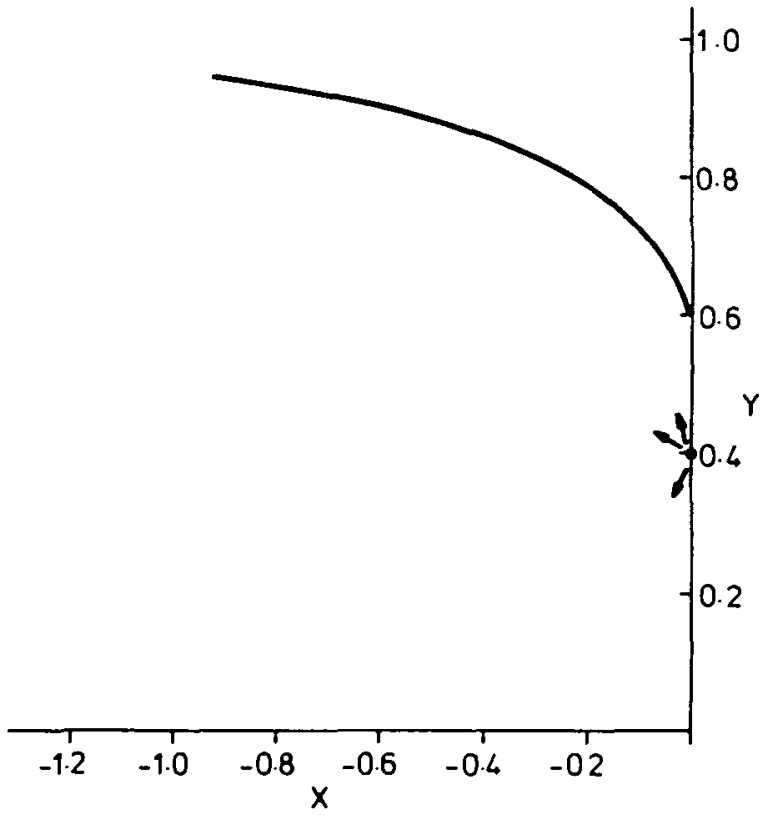

FIGURE 4(b)

FIgURE 4. Flows induced by a source at various positions within a fluid (a) $\delta=0$ (b) $\delta=0.4$ (c) $\delta=0.8$ (d) $\delta=1.2$. $\Delta=$ Hocking's numerical solution 


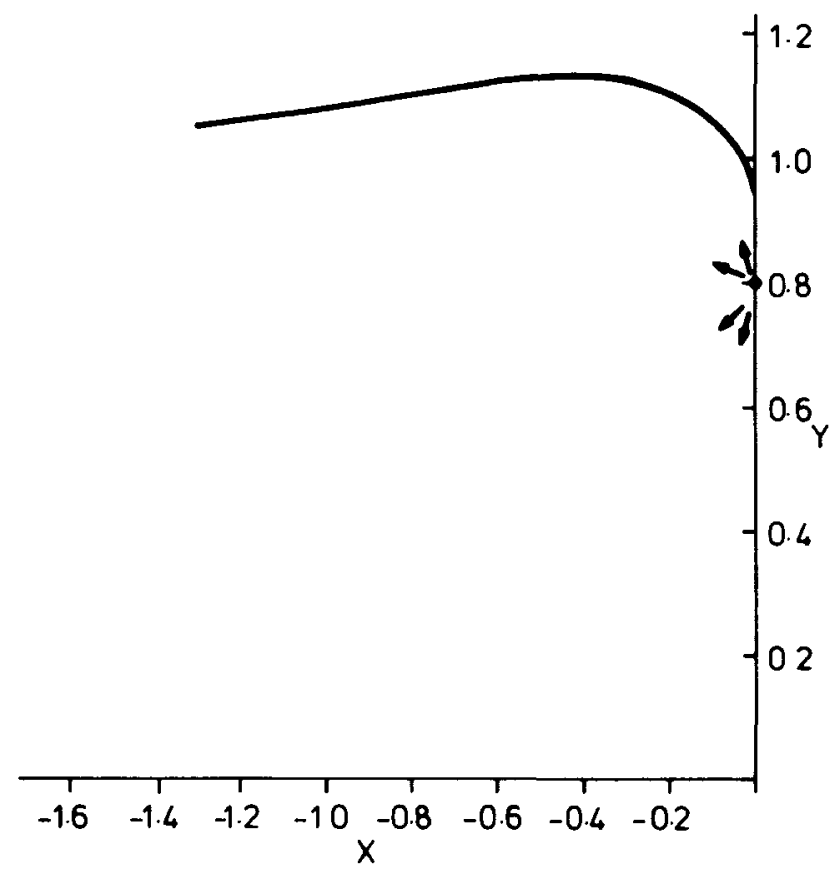

Figure 4(c)

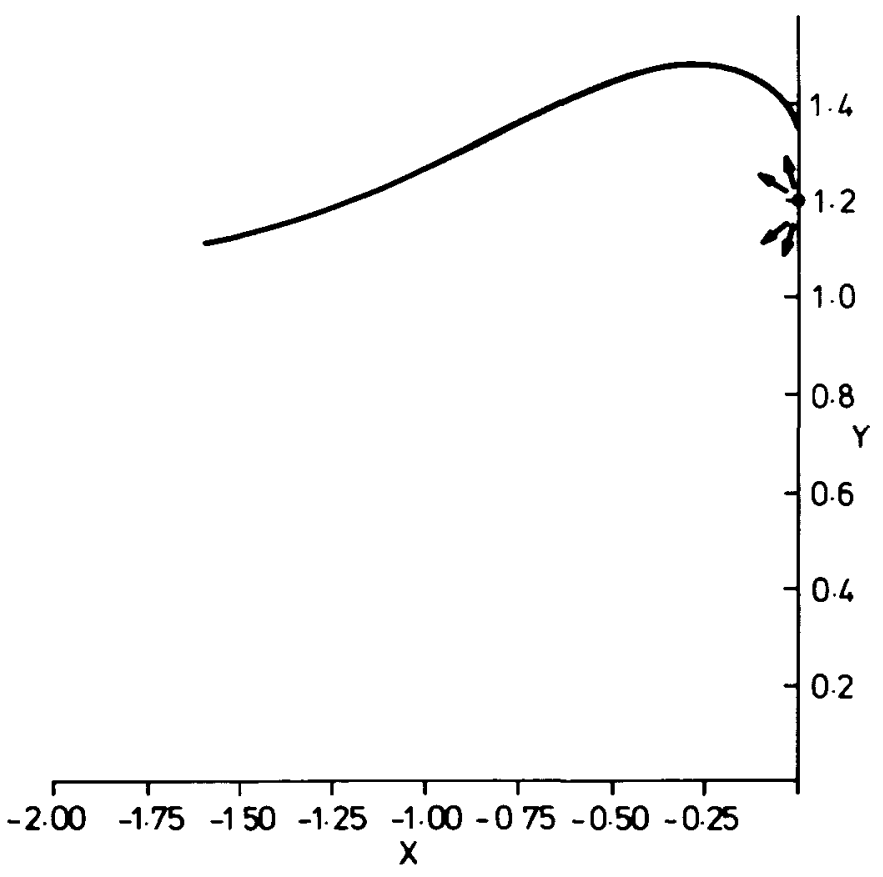

FIGURE 4(d) 


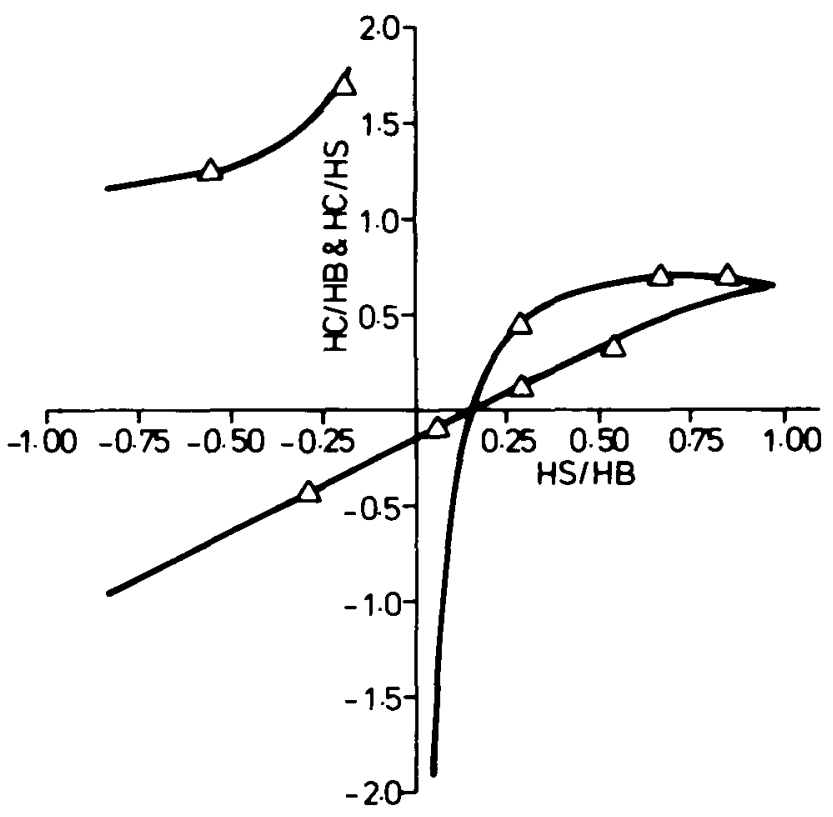

FIGURE 5. Cusp-to-base and cusp-to-source ratios vs source-to-base ratio. $\Delta=$ Hocking's numerical solution

4(a) for the free-surface elevation, and in Figure 5 for the cusp-to-source and cusp-to-base ratios. The agreement between the results is seen to be very good.

\section{Conclusions}

A conformal transformation and integral equation technique has been used to construct solutions to the steady flow induced by a source submerged beneath a cusped free surface and above a flat horizontal bottom when there is no restoring force. Solutions are found at all submergences of the source in a simple closed form, and the numerical and asymptotic results of Collings and Hocking are confirmed.

\section{Acknowledgement}

The authors would like to thank the Editor for bringing to their attention the work of G. C. Hocking [6] in preprint form. 


\section{References}

[1] M. I. G. Bloor, "Large amplitude surface waves", J. Fluid Mech. 84 (1978) 167-179.

[2] M. I. G. Bloor and A. C. King, "Free surface flow over a step", J. Fluid Mech. 182 (1987) 193-208.

[3] 1. L. Collings, "Two infinite-Froude number cusped free-surface flows due to a submerged line source or sink", J. Austral. Math. Soc. Ser. B 28 (1986) 260-270.

[4] T. H. Havelock, "The method of images in some problems of surface waves", Proc. Roy. Soc. London Ser. A 115 (1927) 268-280.

[5] G. C. Hocking, "Cusplike free-surface flows due to a submerged source or sink in the presence of a flat or sloping bottom", J. Austral. Math. Soc. Ser. B 26 (1985) 470-486.

[6] G. C. Hocking, "Infinite Froude number solutions to the problem of a submerged source or sink", J. Austral. Math. Soc. Ser. B 29 (1988) 401-409.

[7] D. H. Peregrine, "A line source beneath a free surface", University of Wisconsin Report 1248 (1972),

[8] E. O. Tuck and J. M. Vanden-Broeck, "A cusplike free-surface flow due to a submerged source or sink", J. Austral. Math. Soc. Ser. B 25 (1984) 443-450.

[9] J. M. Vanden-Broeck and J. M. Keller, "Free surface flow due to a sink", J. Fluid Mech. 175 (1987) 109-117.

[10] J. M. Vanden-Broeck, L. W. Schwartz and E. O. Tuck, "Divergent low Froude number series expansion of non-linear free surface flow problems", Proc. Roy. Soc. London Ser. A 361 (1978) 207-224. 